\section{Butterfly Network}

Butterfly Network has reinvented ultrasound from the ground up through it's pocket-sized device, Butterfly iQ, the first handheld whole-body ultrasound system. Powered by a single silicon chip on a handheld, smartphone-connected device, Butterfly provides a personalized experience for healthcare providers at a drastically lower cost - starting under $\$ 2000$.

For over 50 years, ultrasound machines have used incredibly fragile and expensive piezo crystals, with systems costing upwards of $\$ 50000$, each wired and attached with cables to a separate machine. Those crystals must be tuned to produce three types of ultrasonic waves - linear, curved and phased - requiring separate transducers to capture specific imaging depths to view different parts of the body. Comparatively, Butterfly has replaced these delicate crystals with a single silicon chip. The chip, made from an array of thousands of programmable

micro-machined sensors (you can think of this as a grid of very small drums) is capable of emulating all three ultrasonic wave patterns with a single probe, all on one handheld device. Through this fundamentally new invention, Butterfly is able to drastically lower the cost of its device by leveraging

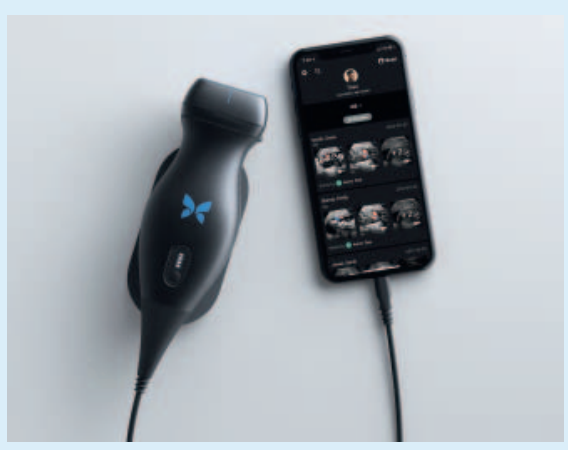

the same manufacturing as consumer products, allowing for high precision, high quality ultrasound to be produced at scale. 
Butterfly iQ was cleared by the FDA for 13 indications, making it the broadest FDA clearance ever for a single ultrasound transducer. The result is a whole-body ultrasound system that is unlike any device on the market.Most recently, Butterfly iQ received CE Mark approval that allows the device to be sold in the UK, Europe, Australia and New Zealand.

At a significantly reduced cost with no drop in quality, Butterfly iQ is making medical imaging universally accessible and affordable. Soon, the newest software updates will also incorporate Al, making it drastically easier to capture a usable image. Through both of these important updates, nurses, EMTs and physician assistants, among many other medical professionals, will now be able to easily and safely administer ultrasound for their patients.

Leveraging an integrated software platform, Butterfly seamlessly connects users to collaborate across the hospital or around the world. Ultrasound studies are uploaded to Butterfly's platform for safe and secure access, storing patient data in a HIPAAcompliant cloud that leverages best-inclass security and encryption.

For almost every disease across every stage of human life, ultrasound can play an im-

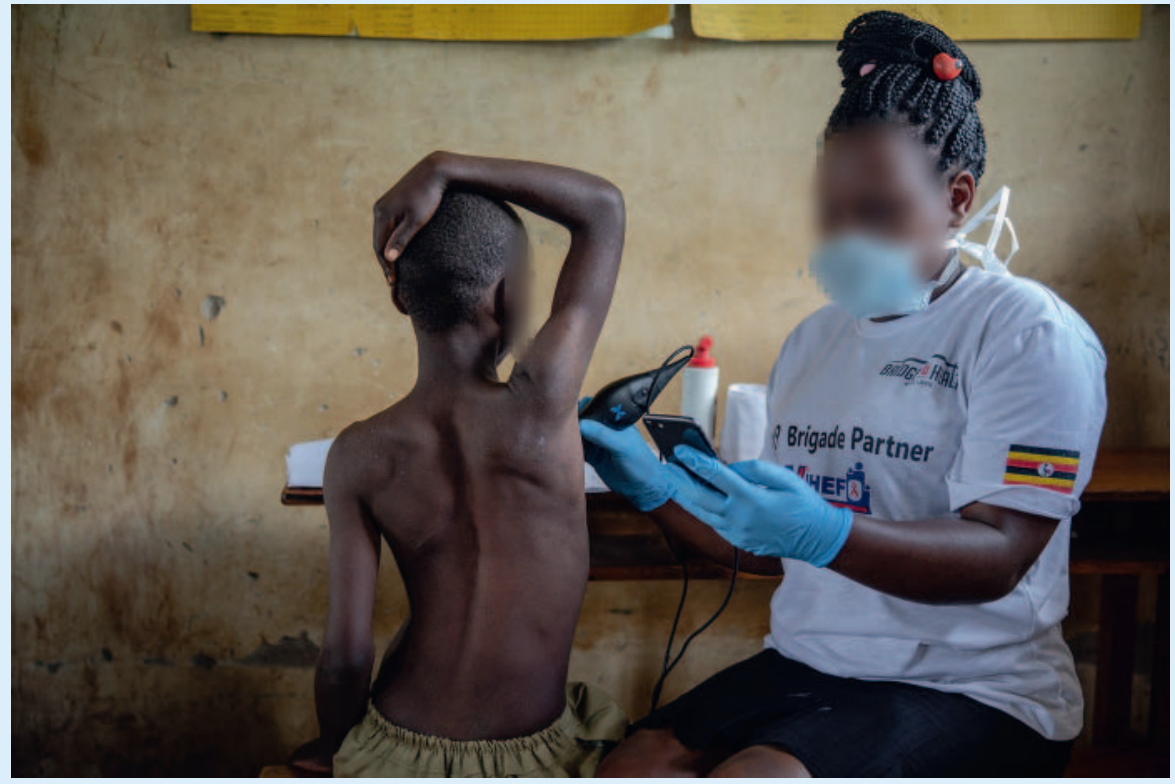

portant role. Butterfly envisions a world where personal ultrasound is just like a glucose meter, blood pressure cuff or a thermometer. Whether you have a chronic disease or a condition that requires regular monitoring, personal ultrasound will be there to find disease in time.

Working with many global health partners, including Bridge to Health, a non-profit that used these devices to help train doctors in Uganda to diagnose pneumonia and other ailments in children and other local residents, Butterfly iQ is helping to save lives in low resource settings around the world. By making medical imaging universally accessible and affordable, Butterfly is democratizing healthcare.

For more information on handheld mobile devices, visit www.efsumb.org. The Use of Handheld Ultrasound Devices - An EFSUMB Position Paper, Michael Bachmann Nielsen 